\title{
DHEA metabolism to the neurosteroid androsterone: a possible mechanism of DHEA's antidepressant action
}

\author{
Rivka Ben Dor ${ }^{1}$, Christine E. Marx², Lawrence J. Shampine ${ }^{2}$, David R. Rubinow ${ }^{3}$, and Peter \\ J. Schmidt ${ }^{1}$ \\ ${ }^{1}$ Behavioral Endocrinology Branch, National Institute of Mental Health, National Institutes of \\ Health, Public Health Service, Department of Health \& Human Services, Building 10, Room \\ 25330, 10 Center Dr MSC 1277, Bethesda, MD 20892-1277, USA \\ ${ }^{2}$ Psychiatry and Behavioral Sciences, Duke University Medical Center, Durham, VA Medical \\ Center, Durham, NC, USA \\ ${ }^{3}$ Department of Psychiatry, University of North Carolina, Chapel Hill, NC, USA
}

\begin{abstract}
Background-Alterations in neurosteroid secretion have been implicated in the efficacy of antidepressants. In a previous study, the adrenal androgen DHEA, a precursor of the neurosteroid androsterone, produced antidepressant and libido-enhancing effects in patients with midlife depression. To investigate the mechanisms underlying DHEA's behavioral effects in this same patient group, we examined plasma levels of four additional neurosteroids implicated in the regulation of affective behavior.
\end{abstract}

Methods-Blood samples were assayed for neurosteroids in men $(n=13)$ and women $(n=10)$ with midlife depression who previously participated in a crossover study in which DHEA and placebo were administered for 6 weeks each. Depression severity was measured by the Center for Epidemiologic Studies Depression Scale (CES-D). Plasma levels of androsterone (ADT), allopregnanolone, pregnanolone, and pregnenolone were measured by GC-MS at baseline and week 6 of each treatment phase. Data were analyzed with repeated measures analysis of variance (ANOVA-R) and Bonferroni $t$ tests.

Results-ADT levels (but not allopregnanolone, pregnanolone, and pregnenolone) increased after DHEA but not after placebo $\left(F_{2,42}=3.3, p<0.05\right)$. Post-DHEA ADT levels were higher in

Correspondence to: Peter J. Schmidt.

Author contribution Drs. Schmidt and Rubinow designed the study and wrote the protocol. Drs. Ben Dor and Schmidt managed the literature searches and analyses. Drs. Ben Dor and Schmdit undertook the statistical analysis, and Dr. Ben Dor wrote the first draft of the manuscript. Dr. Marx conducted neurosteroid quantifications and contributed to the manuscript. All authors contributed to and have approved the final manuscript.

Conflict of interest This work was written as part of Drs. Ben Dor and Schmidt's official duties as Government employees. The views expressed in this article do not necessarily represent the views of the NIMH, NIH, HHS, the Department of Veterans Affairs, or the US Government. This work was supported by the National Institute of Mental Health Intramural Research Program, the VA Mid-Atlantic MIRECC, and a VA Career Development Transition Award. The NIMH had no further role in study design, the collection, analysis, and interpretation of data; in the writing of the report; and in the decision to submit the paper for publication. Dr. Rubinow is a funded member of the Editorial Board for a Servier sponsored journal (Dialogues in Clinical Neuroscience). Dr. Marx is an applicant or coapplicant on pending patent applications focusing on the use of neurosteroids and derivatives for CNS disorders and for lowering cholesterol, no patents issued and no licensing in place (VA 208 waiver in place). None of the other authors have any disclosures to report. 
women than men $\left[t_{63}=2.9, p<0.05\right]$. However, in both men and women who met criteria for clinical response on the CES-D, baseline ADT levels significantly increased post-DHEA, and the magnitude of the ADT increase post-DHEA treatment was similar in men and women. Consequently, it was the non-responders who accounted for the sex difference in post-DHEA plasma ADT levels, a difference that was driven by values in two women (the only female nonresponders).

Conclusions-The small sample size notwithstanding, these data emphasize the potential behavioral relevance of ADT in humans, which may include contribution to the antidepressant effects of DHEA.

\section{Keywords}

DHEA; Androsterone; Depression; Neurosteroids

\section{Introduction}

In both humans and animals, neurosteroids have demonstrated psychotropic action and have been implicated in both the pathogenesis of depression and antidepressant action.

Allopregnanolone and pregnanolone, both metabolites of progesterone, and androsterone, a metabolite of both DHEA and dihydrotestosterone, are potent modulators of the GABA receptor (Majewska et al. 1986; Park-Chung et al. 1999). Although results are not consistent, studies report abnormalities in blood levels of these neurosteroids in depressed men and women (Eser et al. 2006; Kurita et al. 2013) and normalized levels in both plasma and CSF of patients successfully treated with antidepressants (Eser et al. 2006; Romeo et al. 1998; Schule et al. 2006; Schule et al. 2007; Ströhle et al. 1999; Uzunova et al. 1998). Finally, antidepressants promote the activity of synthetic enzymes involved in the formation of neurosteroids (i.e., 3-a-hydroxysteroid dehydrogenase [3a-HSD]), thus favoring the formation of neurosteroids (Griffin and Mellon 1999; Schule et al. 2006).

DHEA has a potential role in the mitigation of stress and the promotion of resilience in humans (Russo et al. 2012). Several clinical trials also have demonstrated positive effects of DHEA therapy under placebo-controlled conditions on mood symptoms in depressive disorders (Maninger et al. 2009), sexual function (Bloch et al. 2013), and well-being (Bloch et al. 2012), albeit in the context of several negative trials in older post-menopausal women involving many of the same outcome measures (Davis et al. 2011). As DHEA acts directly as a neurosteroid and serves as an indirect precursor of several neurosteroid hormones, it is possible that alterations in neurosteroid levels within the CNS could contribute to the antidepressant effects of DHEA described in preliminary reports.

A potential role for the neurosteroid androsterone (ADT) in the mediation of DHEA's therapeutic effects was suggested by Bloch et al. (2006) in his investigation of men in whom hypogonadism was induced with GnRH agonist. In this study, Bloch et al. (2006) observed that changes in CSF levels of ADT (but not of testosterone (T) or other neurosteroids) were correlated with changes in sexual functioning, both during hypogonadism and $\mathrm{T}$ replacement. In rodents, DHEA treatment increases levels of allopregnanolone in both plasma as well as in the hippocampus and hypothalamus (Bernardi et al. 2005). Further, 
several trials of DHEA in men and women have reported a treatment-related increase in plasma levels of allopregnanolone (and progesterone) in men and post-menopausal women (Genazzani et al. 2003; Nadjafi-Triebsch et al. 2003; Stomati et al. 2000). Finally, pregnenolone, a precursor steroid of both DHEA and progesterone, and its sulfated metabolite pregnenolone-sulfate produce anxiety-like behaviors in rodents, and levels of both pregnenolone and pregnenolone sulfate in mouse brain are decreased after DHEA administration (Melchior and Ritzmann 1994).

In a previous randomized controlled trial of men and women with midlife-onset depression, we observed that treatment with the adrenal androgen DHEA enhances mood and sexual functioning. This earlier study, however, failed to identify a specific relationship between plasma levels of DHEA or any other measured hormone and improvements in behavior and mood. Although DHEA treatment also increased levels of testosterone (T) and androstenedione in both men and women, these changes were not associated with the response to DHEA (Schmidt et al. 2005).

In the current study, we examined the possibility that DHEA's psychotropic effects in midlife-onset depression could be mediated by its conversion to a neurosteroid metabolite or by its upstream actions on enzymes involved in the formation of other neurosteroid metabolites. We were interested in measuring the 3a,5a-reduced metabolite of DHEA, ADT, which is a potent modulator of the $\mathrm{GABA}_{\mathrm{A}}$ receptor (Majewska 1992; Park-Chung et al. 1999). The aim of this study was to investigate if plasma levels of ADT, allopregnanolone, pregnanolone, and pregnenolone are altered by oral DHEA administration in men and women with midlife-onset depression. Additionally, despite the limitations of the small sample size, we explored possible relationships between the four targeted neurosteroid plasma levels and changes in mood in these men and women.

\section{Experimental procedures}

\section{Participants}

We analyzed plasma levels of ADT, allopregnanolone, pregnanolone, and pregnenolone in men and women with midlife-onset depression who participated in a randomized controlled study, conducted in 1996-2002, at the National Institute of Mental Health (NIMH) Midlife Clinic in Bethesda, MD. Participants were men and women aged 40-65 years who reported the onset of depression within the past 5 years (and who were symptom free for at least 2 years before the onset of their current episode of depression). Reproductive status was recorded in women but was not a selection criterion for the original treatment trial. All men and women met structured diagnostic criteria for major or minor depression (the criteria for minor depression are identical to those of major depression with the exception that subjects do not report the full five symptoms required for major depression but must report and meet criteria with at least two symptoms) (Table 1). Twenty-three of the 46 participants who completed the original study (i.e., $50 \%$ of the original sample) were included in this study. Inclusion criteria consisted of the availability of plasma samples at each of the three time points in the study (i.e., baseline [pretreatment] and 6 weeks after both DHEA and placebo treatments) and, in women, those who also met the reproductive inclusion criteria (i.e., those women who were peri- or post-menopausal or in whom all blood samples were obtained 
during the follicular phase of the menstrual cycle). In women, luteal blood samples were excluded because high progesterone levels during the luteal phase would increase plasma levels of allopregnanolone and pregnanolone and complicate comparisons by sex or stage of reproductive life. Details regarding eligibility, recruitment, and study design can be found in the original study (Schmidt et al. 2005) and are briefly described below. This study was approved by the Intramural Review Board of the NIMH. Before participating in the study, all subjects provided oral and written informed consent.

\section{Study design}

In the original RCT, participants were randomly assigned to one of two groups defined by the order in which DHEA and placebo were administered. Each treatment phase lasted 6 weeks, with 1-2 weeks of washout before crossover. Patients were treated with DHEA (obtained from Diosynth Biotechnology, Chicago, IL, and National Biochemicals Corporation, Twinsburg, OH), $30 \mathrm{mg}$ three times a day for 3 weeks, followed by $150 \mathrm{mg}$ three times a day for 3 weeks, for a total of 6 weeks. Placebo treatment was also administered for six weeks. Clinic visits during which symptom ratings were completed and blood samples obtained occurred after 3 and 6 weeks of each treatment. Blood samples were obtained prior to $10 \mathrm{a} . \mathrm{m}$. Data for this study were derived from the baseline (pretreatment) and week 6 visits only.

\section{Outcome measures}

\section{Targeted neurosteroid and hormone assays}

Blood samples were drawn at pretreatment baseline, at the end of treatment with DHEA, and at the end of treatment with placebo. Plasma was kept frozen at $-80{ }^{\circ} \mathrm{C}$ until it was analyzed by gas chromatography/mass spectrometry preceded by high-performance liquid chromatography for ADT, allopregnanolone, pregnanolone, and pregnenolone quantifications.

Quantification of ADT, allopregnanolone, pregnanolone, and pregnenolone was performed by gas chromatography/mass spectrometry (GC/MS) preceded by high-performance liquid chromatography (HPLC), as previously described with modifications (Marx et al. 2006b; Marx et al. 2006c; Naylor et al. 2008; Sripada et al. 2013; Sripada et al. 2014). HPLC purification was performed on an 1100 Series Agilent instrument. Standards and samples were then derivatized using heptafluorobutyric acid anhydride (HFBA) injected onto an Agilent 5973 mass spectrometer (MS) coupled to an Agilent $6890 \mathrm{~N}$-gas chromatograph (GC) equipped with an Agilent HP-5MS $30 \mathrm{~m} \times 0.250 \mathrm{~mm} \times 0.25 \mu \mathrm{m}$ capillary column. Positive ion electron impact (EI) ionization was used in the GC/MS component, and in positive ion EI mode, samples were analyzed in duplicate using helium as the carrier gas. Deuterated allopregnanolone (D4-allopregnanolone) was utilized as the internal standard for allopregnanolone, pregnanolone, and ADT quantifications; deuterated pregnenolone (D4pregnenolone) was utilized for pregnenolone quantifications (Cambridge Isotope Laboratories, Inc. Andover, MA). For neurosteroid quantification, the standard curve for the steroid of interest was prepared by combining varying known quantities of the neurosteroid (Steraloids, Newport, RI) with a constant amount of deuterated internal standard. Identically 
to the samples, the standard curve was extracted three times in ethyl acetate prior to HPLC purification and GC/MS injection (standard curve $r^{2}=0.99$ for each neurosteroid). The area under the peak of each known quantity of neurosteroid was divided by the area under the peak of the internal standard. This ratio was then plotted on the $y$-axis against known quantities of each steroid to generate the standard curve. In addition to identifying each neurosteroid's characteristic GC/MS retention time, we were also able to identify a unique mass fragmentation pattern — a definitive structural identifier—for each neurosteroid. Mass spectrometer singleion monitoring (selected ion monitoring (SIM)) was used to focus on the most abundant ion fragment for each HFBA derivative. The mass to charge ratios of the most intense ion fragments in SIM mode for each of the compounds are as follows:

$\mathrm{ADT}=486.2$, allopregnanolone $=496.2$, pregnenolone $=496.2$, and pregnanolone $=298.2$. Only peaks with a signal to noise ratio $25: 1$ were integrated. The limit of neurosteroid quantification with this methodology was $2 \mathrm{pg}$ for ADT, allopregnanolone, and pregnanolone and $10 \mathrm{pg}$ for pregnenolone. Intra-assay coefficients of variation were $1.1 \%$ for ADT, $3.2 \%$ for allopregnanolone, $1.3 \%$ for pregnanolone, and $1.5 \%$ for pregnenolone.

Blood samples for DHEA, DHEAS, estradiol (E2), total and free T, and androstenedione were assayed by immunoassay under batched conditions, with samples from the same person run within the same batch at the Mayo Clinic Laboratories in Rochester, MN, or at the Endocrine Sciences Laboratories in Calabasas Hills, CA. Inter-assay coefficients of variation $(\mathrm{CVs})$ in the range of the measured values were as follows (CVs from Mayo Clinic Laboratories appear in parentheses): DHEA $=(7.0 \%) 8.4 \%$, DHEAS $=(8.0 \%) 8.4 \%$, $\mathrm{E} 2=(10 \%) 9.2 \%$, total and free $\mathrm{T}$ (calculated $)=(9.5 \%) 8.9 \%$, and androstenedione $=9.1 \%$. The measures of total $\mathrm{T}$ and free $\mathrm{T}$ were performed on serum samples, whereas the remaining hormone levels were measured in plasma.

Plasma levels of ADT, allopregnanolone, pregnanolone, and pregnenolone were obtained from samples collected in the original study to permit determination of the correlation with earlier obtained steroids (DHEA(S), T, free T, androstenedione and E2) and the clinical states observed (i.e., depressed vs non-depressed) (Schmidt et al. 2005).

\section{Depression rating scale}

Severity of depression was assessed using the Center for Epidemiologic Studies Depression Scale (CES-D) (Radloff 1977), one of the primary outcome measures employed in the previous trial.

\section{Statistical analysis}

Analysis of variance was performed with repeated measures (repeated measures analysis of variance (ANOVA-R)) (Systat 10.0 [SPSS Inc., Chicago, IL]), with the within-subject variable treatment condition (baseline vs. DHEA or placebo) and the between-subjects variable sex. Post hoc testing was performed with Bonferroni $t$ tests.

Since we identified that plasma levels of ADT (but not those of the other neurosteroids measured) increased after DHEA treatment, we then examined the relationship between plasma ADT levels and mood responses to DHEA treatment. A positive mood response to 
DHEA (i.e., responder) was defined by a $50 \%$ or greater decrease in CES-D scores relative to pretreatment baseline. We performed ANOVA-Rs on both raw and log-transformed plasma ADT levels to evaluate the association between plasma ADT levels and CES-D scores. The within-subject variable was treatment condition (baseline vs. DHEA or placebo), and the between-subjects variables were sex and response to treatment.

Our previous study failed to identify any association between plasma levels of DHEA, DHEAS, total T, free T, and DHEA-induced changes in mood or sexual function (Schmidt et al. 2005). In order to test these original findings in the subset of the original sample included in the current study, we performed ANOVA-Rs for levels of DHEA, DHEAS, free T, total T, androstenedione, and estradiol, with the within-subject variable treatment condition (baseline vs. DHEA or placebo) and the between-subjects variable sex.

\section{Results}

\section{Subject characteristics}

Plasma samples were available in 23 of the 46 participants who completed the original study (see "Experimental procedures" section) Plasma levels of ADT, allopregnanolone, pregnanolone, and pregnenolone at baseline and after 6 weeks of DHEA or placebo treatment were analyzed in 13 men ranging in age from 42 to 63 years (mean=52.3 \pm 7.5 years SD) and 10 women ranging in age from 43 to 57 years (mean=50.5 \pm 4.4 years SD). Nine women in the study were post-menopausal or peri-menopausal with amenorrhea, and in the one premenopausal subject, blood for evaluation was drawn during the follicular phase only. Six men and eight women met the criteria for current major depression. All other subjects met criteria for current minor depression (Table 1). The subsamples included in this present study were similar to the original sample with respect to treatment response to DHEA as well as baseline and post-treatment hormone measures.

Neurosteroids and hormonal measures-Consistent with our previous report in a larger sample (Schmidt et al. 2005), ANOVA-R showed a significant main effect of condition, reflecting an increase in plasma levels of DHEA, DHEAS, androstenedione, and free $T$ after DHEA treatment compared with baseline and placebo (Table 2). Additionally, ANOVA-R showed significant main effects of sex for plasma levels of free $\mathrm{T}$ and total $\mathrm{T}$ $\left(F_{1,19}=16.9\right.$ and 59.9, $p<0.01$, respectively), reflecting the higher plasma levels of free $\mathrm{T}$ and total $\mathrm{T}$ in men compared with women. (Table 2)

Compared with both baseline and placebo, DHEA treatment significantly increased plasma levels of ADT (ANOVA-R [effect of drug condition] $F_{2,42}=36.1, p<0.01$ ). ADT levels at week 6 of DHEA treatment (but not during either baseline or placebo) were significantly higher in women $(1301.0 \mathrm{pg} / \mathrm{mL})$ than in men $(796.7 \mathrm{pg} / \mathrm{mL})$, ANOVA-R [drug condition $\times$ sex] $F_{2,42}=3.3, p<0.05, t_{63}=2.9, p<0.05$ ) (Table 2 ). Log-transformed ADT values similarly showed a significant drug condition $\times$ sex interaction $(p=0.02)$.

Neither BMIs nor plasma DHEA levels differed significantly between men and women (Table 1), nor were significant correlations observed between BMI and plasma ADT levels $\left(r^{2}=-0.14, p=\mathrm{ns}\right)$ or between BMI and plasma DHEA levels $\left(r^{2}=-0.15, p=\mathrm{ns}\right)$. Finally, the 
increase in DHEA(S) blood levels after DHEA administration compared with baseline levels did not differ between men and women $\left(t_{21}=0.8\right.$ and 0.6 , respectively, $\left.p=\mathrm{ns}\right)$.

There were no significant correlations between plasma ADT levels at week 6 of DHEA treatment and any hormone measured, with the exception of a significant correlation between the change in plasma ADT from baseline to DHEA treatment and the corresponding change in plasma DHEA levels $\left(r^{2}=0.66, p<0.05\right)$. However, this correlation did not remain significant after post hoc Bonferroni adjustment for multiple ( $n=8)$ correlations.

In contrast to ADT, plasma levels of allopregnanolone, pregnanolone, and pregnenolone showed no significant effects of treatment condition alone or any interactive effects of treatment condition and sex (Table 2). ANOVA-R showed a significant sex effect for plasma levels of allopregnanolone (ANOVA-R [sex effect $]=F_{1,21}=6.7, p=0.02$ ), reflecting the higher plasma levels of allopregnanolone observed in women at all sampling points (baseline and after both DHEA and placebo).

\section{ADT plasma levels and behavior}

Consistent with results obtained in our earlier study (Schmidt et al. 2005), the scores of the CES-D significantly improved at week 6 of DHEA treatment compared with baseline and placebo conditions (Fig. 1). Thus, the treatment response characteristics of this subsample were representative of the larger original sample.

Eight of the ten women and seven of the 13 men met CESD criterion for response (i.e., $50 \%$ or greater decrease in CESD scores relative to pretreatment baseline). We observed a significant interaction among drug condition, sex, and mood response $\left(F_{1,19}=7.1, p=0.02\right)$ which reflected the following: In men and women, plasma ADT levels in responders increased significantly from baseline to the end of DHEA treatment $\left(t_{19}=4.4, p<0.01\right.$ for both men and women) (Fig. 2), whereas there was no significant difference in post-DHEA plasma ADT levels between men and women responders $\left(t_{38}=0.03, p=n s\right)$. Thus, both baseline and post-DHEA treatment levels of ADT were similar in men and women responders. In contrast, non-responders (men $[n=6]$ and women $[n=2]$ ) showed a different pattern of change in plasma ADT levels. Non-responding men did not show a significant increase in ADT (plasma ADT levels at baseline compared with week 6 post-DHEA; $t_{19}=1.2, p=n s$ ), whereas non-responding women showed a greater increase than women responders (women responders compared with non-responders; $t_{38}=3.0, p<0.05$ ) (Fig. 2).

\section{Discussion}

This study yielded two main findings: (1) administered daily, high-dose (450 mg) oral DHEA significantly increases plasma levels of ADT but not allopregnanolone, pregnanolone, and pregnenolone and (2) the antidepressant effects of DHEA are accompanied by a significant increase in plasma ADT levels in both men and women. Notwithstanding the small sample and the presence of men and women with both major and minor depression, the magnitude of the changes in plasma ADT levels after DHEA administration was similar in both men and women whose mood symptoms responded to 
DHEA, whereas men and women who did not meet criteria for response showed divergent patterns of post-DHEA treatment ADT levels.

Despite evidence that DHEA can modulate mood and behavior, the physiologic and therapeutic roles of DHEA are as yet fully characterized. Although some trials demonstrate therapeutic effects of administered DHEA in several behavioral conditions (Maninger et al. 2009), other trials find no benefit (Davis et al. 2011). The absence of a specific mechanism that mediates DHEA's physiologic (or therapeutic) actions contributes to controversy surrounding the identification of a specific role for this hormone. In contrast to other steroid hormones, DHEA does not bind to a parent receptor, although some binding to the androgen receptor has been reported (Lu et al. 2003). A wide range of possible mechanisms have been proposed to explain the actions of DHEA in humans (Maninger et al. 2009; Sripada et al.

2013; Sripada et al. 2014) including the following: serving as a precursor hormone to the sex steroids estradiol and testosterone; its binding as a neurosteroid to sigma receptors (Debonnel et al. 1996); its conversion to neurosteroid metabolites active at the GABA receptor (Park-Chung et al. 1999); its role as an antiglucocorticoid (Kalimi et al. 1994; McNelis et al. 2013); or more recently, its conversion to ADIOL, a ligand for estrogen receptor beta, which functions in the CNS to suppress inflammatory responses in both microglia and astrocytes (Saijo et al. 2011). Our data suggest that the neurosteroid metabolite of DHEA, ADT, should be added to the list of potential mediators of DHEA's observed effects on mood.

Changes in plasma levels of ADT accompanied improvements in mood. In men and women who met CES-D criterion for response to DHEA, we observed similar significant increases in plasma ADT levels from baseline to the end of DHEA treatment. Non-responding men showed an attenuated elevation of plasma ADT levels despite receiving the same dosage of DHEA. Paradoxically, women who failed to show a mood response to DHEA showed an exaggerated ADT response to DHEA, but there were only two female non-responders. An abnormal ADT response to DHEA could suggest a difference in HPA pathophysiology relevant to antidepressant responsivity in depression (e.g., abnormalities of $3 \beta$-HSD, 5areductase, and/or 3a-HSD or other adrenocortical steroidogenic pathway pathophysiology). Clearly, a study with a larger sample of responders and non-responders of both sexes is needed. As an additional caveat, although non-polar steroid hormones (e.g., non-sulfated hormones) freely diffuse across the blood-brain barrier, the measurement of peripheral blood levels cannot necessarily be assumed to reflect brain tissue levels of these steroids due to both the de novo synthesis of neurosteroids within the brain and possible tissueconcentrating effects within the CNS. More research is needed to definitively answer the question of neurosteroid compartmentalization in plasma and brain, but initial evidence suggests potential relationships. For example, levels of DHEA in cerebrospinal fluid are correlated with temporal cortex levels in humans (Naylor et al. 2008), and rodent pregnenolone levels in serum are closely correlated with hippocampal pregnenolone levels (Marx et al. 2006a). However, it is possible that some of the observed effects of DHEA on the plasma measures of allopregnanolone, pregnanolone, and pregnenolone do not accurately reflect the effects of DHEA on the brain. Finally, several potentially behaviorally relevant metabolites of DHEA were not measured in this study (e.g., androstenediol), and it 
is possible that these steroids, like ADT, could have contributed to the observed mood improvements observed after DHEA.

ADT, like other sex steroids, can exert its effect on the central nervous system through several possible mechanisms. It is a weak androgen with a lower affinity for the androgen receptor than either DHT or T but with possibly a greater affinity than DHEA (Liao et al. 1973). As noted above, ADT and its sulfate are also potent neurosteroids (Majewska 1992; Park-Chung et al. 1999) that allosterically modulate activity at the $\mathrm{GABA}_{\mathrm{A}}$ receptor complex (albeit potentially in different directions (Park-Chung et al. 1999)) with an affinity comparable to the neurosteroid allopregnanolone (Park-Chung et al. 1999). Studies in lower animals have demonstrated that ADT increases chloride influx activated by $\mathrm{GABA}_{\mathrm{A}}$, with brain region-specific potentiation in the amygdala and hippocampus (Wilson and Biscardi 1997).

Current evidence suggests that circulating ADT levels are derived from the conversion of androgens in peripheral tissues (Luu-The and Labrie 2010). In this study, we observed a significant increase in plasma ADT levels in both men and women after oral DHEA administration. Our findings are consistent with several studies employing radio-labeled steroids that identified DHEA(S) as the principal precursor of ADT (Lindberg et al. 1966; Roberts et al. 1961). Our data are also consistent with later reports of significantly elevated plasma ADT (or its glucuronide) and/or androstenedione after the administration of DHEA, either by oral or by transdermal routes (Bloch et al. 2013; Hunt et al. 2000; Kudielka et al. 1998; Labrie et al. 2009; Morales et al. 1998).

DHEA treatment had no effect on plasma levels of allopregnanolone, pregnanolone, and pregnenolone. We did observe a sex difference in plasma allopregnanolone (but not in plasma pregnanolone or pregnenolone) levels, with women having higher levels compared with men even at baseline prior to receiving treatment. A previous study by Genazzani et al. (1998) observed an age-related decline in plasma allopregnanolone levels in men that was absent in post-menopausal women. Thus, the age of our men and women could contribute to the observed sex difference in allopregnanolone levels. Unfortunately, plasma progesterone levels were measured in neither men nor women, but it is possible that those women who were premenopausal and peri-menopausal had sufficient ovarian function to secrete progesterone, thereby contributing to elevated allopregnanolone levels in the women (Schmidt et al. 1994).

Our failure to observe an increase in plasma allopregnanolone (or pregnanolone) levels after DHEA treatment could reflect the relatively brief duration of treatment in this study, as well as differences in assay methods. The studies reporting a DHEA-related increase in allopregnanolone involved the administration of DHEA for several months, whereas in this study, DHEA was administered for 6 weeks only. Additionally, in contrast to those studies reporting increases in allopregnanolone, we employed GC/MS techniques and not RIA methods (Bernardi et al. 2005; Genazzani et al. 2003). Nonetheless, we observed clinical improvements in symptoms and elevated plasma levels of ADT but not allopregnanolone and pregnanolone after 6 weeks of treatment. Finally, despite evidence in rodents that DHEA lowers CNS levels of the anxiogenic neurosteroid, pregnenolone (Melchior and Ritzmann 
1994), we observed no evidence suggesting that DHEA's antidepressant effects were mediated by alterations in pregnenolone secretion.

Despite several limitations, the current study emphasizes the potential importance of the neurosteroid ADT for the behavioral effects of DHEA. The association between CSFADT levels and improved male sexual functioning, reported in an earlier study (Bloch et al. 2006), further supports ADT's behavioral relevance. Our data suggest that the putative psychotropic effects of androgens, including DHEA, may, in fact, reflect changing levels of ADT, which would make it a key mechanism in mood and a worthy subject for future research.

\section{References}

Bernardi F, Casarosa E, Pluchino N, Palumbo M, Genazzani AD, Luisi S, Genazzani AR (2005) Effect of dehydroepiandrosterone on central and peripheral levels of allopregnanolone and $\beta$-endorphin. Fertil Steril 83(Suppl 1):1161-1168 [PubMed: 15831289]

Bloch M, Ish-Shalom S, Greenman Y, Klein E, Latzer Y (2012) Dehydroepiandrosterone treatment effects on weight, bone density, bone metabolism and mood in women suffering from anorexia nervosa-a pilot study. Psychiatry Res 200:544-549 [PubMed: 22858403]

Bloch M, Meiboom H, Zaig I, Schreiber S, Abramov L (2013) The use of dehydroepiandrosterone in the treatment of hypoactive sexual desire disorder: a report of gender differences. Eur Neuropsychopharmacol 23:910-918 [PubMed: 23084789]

Bloch M, Rubinow DR, Berlin K, Kevala KR, Kim HY, Schmidt PJ (2006) Monoamines and neurosteroids in sexual function during induced hypogonadism in healthy men. Arch Gen Psychiatr 63: 450-456 [PubMed: 16585475]

Davis SR, Panjari M, Stanczyk FZ (2011) Clinical review: DHEA replacement for postmenopausal women. J Clin Endocrinol Metab 96:1642-1653 [PubMed: 21411558]

Debonnel G, Bergeron R, de Montigny C (1996) Potentiation by dehydroepiandrosterone of the neuronal response to $\mathrm{N}$-methyl-D-aspartate in the $\mathrm{CA}_{3}$ region of the rat dorsal hippocampus: an effect mediated via sigma receptors. J Endocrinol 150:S33-S42 [PubMed: 8943785]

Eser D, Schüle C, Baghai TC, Romeo E, Uzunov DP, Rupprecht R (2006) Neuroactive steroids and affective disorders. Pharmacol Biochem Behav 84:656-666 [PubMed: 16831459]

Genazzani AD, Stomati M, Bernardi F, Pieri M, Rovati L, Genazzani AR (2003) Long-term low-dose dehydroepiandrosterone oral supplementation in early and late postmenopausal women modulates endocrine parameters and synthesis of neuroactive steroids. Fertil Steril 80:1495-1501 [PubMed: 14667889]

Genazzani AR, Petraglia F, Bernardi F, Casarosa E, Salvestroni C, Tonetti A, Nappi RE, Luisi S, Palumbo M, Purdy RH, Luisi M (1998) Circulating levels of allopregnanolone in humans: gender, age, and endocrine influences. J Clin Exp Neuropsychol 83:2099-2103

Griffin LD, Mellon SH (1999) Selective serotonin reuptake inhibitors directly alter activity of neurosteroidogenic enzymes. Proc Natl Acad Sci U S A 96:13512-13517 [PubMed: 10557352]

Hunt PJ, Gurnell EM, Huppert FA, Richards C, Prevost AT, Wass JAH, Herbert J, Chatterjee VKK (2000) Improvement in mood and fatigue after dehydroepiandrosterone replacement in Addison's disease in a randomized, double blind trial. J Clin Endocrinol Metab 85:4650-4656 [PubMed: 11134123]

Kalimi M, Shafagoj Y, Loria R, Padgett D, Regelson W (1994) Anti-glucocorticoid effects of dehydroepiandrosterone (DHEA). Mol Cell Biochem 131:99-104 [PubMed: 8035785]

Kudielka BM, Hellhammer J, Hellhammer DH, Wolf OT, Pirke K-M, Varadi E, Pilz J, Kirschbaum C (1998) Sex differences in endocrine and psychological responses to psychosocial stress in healthy elderly subjects and the impact of a 2-week dehydroepiandrosterone treatment. J Clin Endocrinol Metab 83:1756-1761 [PubMed: 9589688]

Kurita H, Maeshima H, Kida S, Matsuzaka H, Shimano T, Nakano Y, Baba H, Suzuki T, Arai H (2013) Serum dehydroepiandrosterone (DHEA) and DHEA-sulfate (S) levels in medicated patients with 
major depressive disorder compared with controls. J Affect Disord 146:205-21 [PubMed: 23102506]

Labrie F, Archer D, Bouchard C, Fortier M, Cusan L, Gomez J-L, Girard G, Baron M, Ayotte N, Moreau M, Dube R, Cote I, Labrie C, Lavoie L, Berube R, Belanger P, Berger L, Gilbert L, Martel C, Balser J (2009) Serum steroid levels during 12-week intravaginal dehydroepiandrosterone administration. Menopause 16:897-906 [PubMed: 19436226]

Liao S, Liang T, Fang S, Castaneda E, Shao T-C (1973) Steroid structure and androgenic activity; specificities involved in the receptor binding and nuclear retention of various androgens. J Biol Chem 248: 6154-6162 [PubMed: 4353633]

Lindberg MC, McClenaghan C, Herrmannn WL (1966) Fate of injected dehydroisoandrosterone, testosterone, and dehydroiisoandrosterone sulfate in plasma in the human. Am J Obstet Gynecol 95:743-746 [PubMed: 4223125]

Lu SF, Mo Q, Hu S, Garippa C, Simon NG (2003) Dehydroepiandrosterone upregulates neural androgen receptor level and transcriptional activity. J Neurobiol 57:163-171 [PubMed: 14556282]

Luu-The V, Labrie F (2010) The intracrine sex steroid biosynthesis pathways. Prog Brain Res 181:177-192 [PubMed: 20478438]

Majewska MD (1992) Neurosteroids: endogenous bimodal modulators of the GABAA receptor: mechanism of action and physiological significance. Prog Neurobiol 38:379-395 [PubMed: 1349441]

Majewska MD, Harrison NL, Schwartz RD, Barker JL, Paul SM (1986) Steroid hormone metabolites are barbiturate-like modulators of the GABA receptor. Science 232:1004-1007 [PubMed: 2422758]

Maninger N, Wolkowitz OM, Reus VI, Epel ES, Mellon SH (2009) Neurobiological and neuropsychiatric effects of dehydroepiandrosterone (DHEA) and DHEA sulfate (DHEAS). Front Neuroendocrinol 30:65-91 [PubMed: 19063914]

Marx CE, Shampine LJ, Duncan GE, VanDoren MJ, Grobin AC, Massing MW, Madison RD, Bradford DW, Butterfield MI, Lieberman JA, Morrow AL (2006a) Clozapine markedly elevates pregnenolone in rat hippocampus, cerebral cortex, and serum: candidate mechanism for superior efficacy? Pharmacol Biochem Behav 84:598-608 [PubMed: 16962649]

Marx CE, Trost WT, Shampine L, Behm FM, Giordano LA, Massing MW, Rose JE (2006b) Neuroactive steroids, negative affect, and nicotine dependence severity in male smokers. Psychopharmacology (Berl) 186:462-472 [PubMed: 16402195]

Marx CE, Trost WT, Shampine LJ, Stevens RD, Hulette CM, Steffens DC, Ervin JF, Butterfield MI, Blazer DG, Massing MW, Lieberman JA (2006c) The neurosteroid allopregnanolone is reduced in pre-frontal cortex in Alzheimer's disease. Biol Psychiatr 60:1287-1294

McNelis JC, Manolopoulos KN, Gathercole LL, Bujalska IJ, Stewart PM, Tomlinson JW, Arlt W (2013) Dehydroepiandrosterone exerts antiglucocorticoid action on human preadipocyte proliferation, differentiation, and glucose uptake. Am J Physiol Endocrinol Metab 305:1134-1144

Melchior CL, Ritzmann RF (1994) Pregnenolone and pregnenolone sul-fate, alone and with ethanol, in mice on the plus-maze. Pharmacol Biochem Behav 48:893-897 [PubMed: 7972293]

Morales AJ, Haubricht RH, Hwang JY, Asakura H, Yen SSC (1998) The effect of six months treatment with a $100 \mathrm{mg}$ daily dose of dehydroepiandrosterone (DHEA) on circulating sex steroids, body composition and muscle strength in age-advanced men and women. Clin Endocrinol 49:421-432

Nadjafi-Triebsch C, Huell M, Burki D, Rohr UD (2003) Progesterone increase under DHEAsubstitution in males. Maturitas 45:231-235 [PubMed: 12818469]

Naylor JC, Hulette CM, Steffens DC, Shampine LJ, Ervin JF, Payne VM, Massing MW, Kilts JD, Strauss JL, Calhoun PS, Calnaido RP, Blazer DG, Lieberman JA, Madison RD, Marx CE (2008) Cerebrospinal fluid dehydroepiandrosterone levels are correlated with brain dehydroepiandrosterone levels, elevated in Alzheimer's disease, and related to neuropathological disease stage. J Clin Endocrinol Metab 93:3173-3178 [PubMed: 18477662]

Park-Chung M, Malayev A, Purdy RH, Gibbs TT, Farb DH (1999) Sulfated and unsulfated steroids modulate $\gamma$-aminobutyric acid $_{\mathrm{A}}$ receptor function through distinct sites. Brain Res 830:72-87 [PubMed: 10350561] 
Radloff LS (1977) The CES-D scale: a self-report depression scale for research in the general population. Appl Psychol Meas 1: 385-401

Roberts KD, VandeWiele RL, Lieberman S (1961) The conversion in vivo of dehydroisoandrosterone sulfate to androsterone and etiocholanolone glucuronidates. J Biol Chem 236:2213-2215 [PubMed: 13742168]

Romeo E, Strohle A, Spalletta G, di Michele F, Hermann B, Holsboer F, Pasini A, Rupprecht R (1998) Effects of antidepressant treatment on neuroactive steroids in major depression. Am J Psychiatr 155:910-913 [PubMed: 9659856]

Russo SJ, Murrough JW, Han M, Charney DS, Nestler EJ (2012) Neurobiology of resilience. Nat Neurosci 15:1475-1484 [PubMed: 23064380]

Saijo K, Collier JG, Li AC, Katzenellenbogen JA, Glass CK (2011) An ADIOL-ER $\beta-C t B P$ transrepression pathway negatively regulates microglia-mediated inflammation. Cell 145:584-595 [PubMed: 21565615]

Schmidt PJ, Daly RC, Bloch M, Smith MJ, Danaceau MA, Simpson S, Clair L, Murphy JH, Haq NA, Rubinow DR (2005) Dehydroepiandrosterone monotherapy in midlife-onset major and minor depression. Arch Gen Psychiatr 62:154-162 [PubMed: 15699292]

Schmidt PJ, Purdy RH, Moore PH, Jr, Paul SM, Rubinow DR (1994) Circulating levels of anxiolytic steroids in the luteal phase in women with premenstrual syndrome and in control subjects. J Clin Endocrinol Metab 79:1256-1260 [PubMed: 7962316]

Schule C, Baghai TC, di Michele F, Eser D, Pasini A, Schwarz M, Rupprecht R, Romeo E (2007) Effects of combination treatment with mood stabilizers and mirtazapine on plasma concentrations of neuro-active steroids in depressed patients. Psychoneuroendocrinology 32: 669-680 [PubMed: 17560730]

Schule C, Romeo E, Uzunov DP, Eser D, di Michele F, Baghai TC, Pasini A, Schwarz M, Kempter H, Rupprecht R (2006) Influence of mirtazapine on plasma concentrations of neuroactive steroids in major depression and on 3a-hydroxysteroid dehydrogenase activity. Mol Psychiatr 11:261-272

Soules MR, Sherman S, Parrott E, Rebar R, Santoro N, Utian W, Woods N (2001) Executive summary: Stages of Reproductive Aging Workshop (STRAW). Fertil Steril 76:874-878 [PubMed: 11704104]

Sripada RK, Marx CE, King AP, Rajaram N, Garfinkel SN, Abelson JL, Liberzon I (2013) DHEA enhances emotion regulation neurocircuits and modulates memory for emotional stimuli. Neuropsychopharmacology 38:1798-1807 [PubMed: 23552182]

Sripada RK, Welsh RC, Marx CE, Liberzon I (2014) The neurosteroids allopregnanolone and dehydroepiandrosterone modulate resting-state amygdala connectivity. Hum Brain Mapp 35:32493261 [PubMed: 24302681]

Stomati M, Monteleone P, Casarosa E, Quirici B, Puccetti S, Bernardi F, Genazzani AD, Rovati L, Luisi M, Genazzani AR (2000) Six-month oral dehydroepiandrosterone supplementation in early and late post-menopause. Gynecol Endocrinol 14:342-363 [PubMed: 11109974]

Ströhle A, Romeo E, Hermann B, Pasini A, Spalletta G, di Michele F, Holsboer F, Rupprecht R (1999) Concentrations of $3 a$-reduced neuroactive steroids and their precursors in plasma of patients with major depression and after clinical recovery. Biol Psychiatr 45: 274-277

Uzunova V, Sheline Y, Davis JM, Rasmusson A, Uzunov DP, Costa E, Guidotti A (1998) Increase in the cerebrospinal fluid content of neurosteroids in patients with unipolar major depression who are receiving fluoxetine or fluvoxamine. Proc Natl Acad Sci U S A 95:3239-3244 [PubMed: 9501247]

Wilson MA, Biscardi R (1997) Influence of gender and brain region on neurosteroid modulation of GABA responses in rats. Life Sci 60: 1679-1691 [PubMed: 9129123]

Psychopharmacology (Berl). Author manuscript; available in PMC 2018 December 28. 

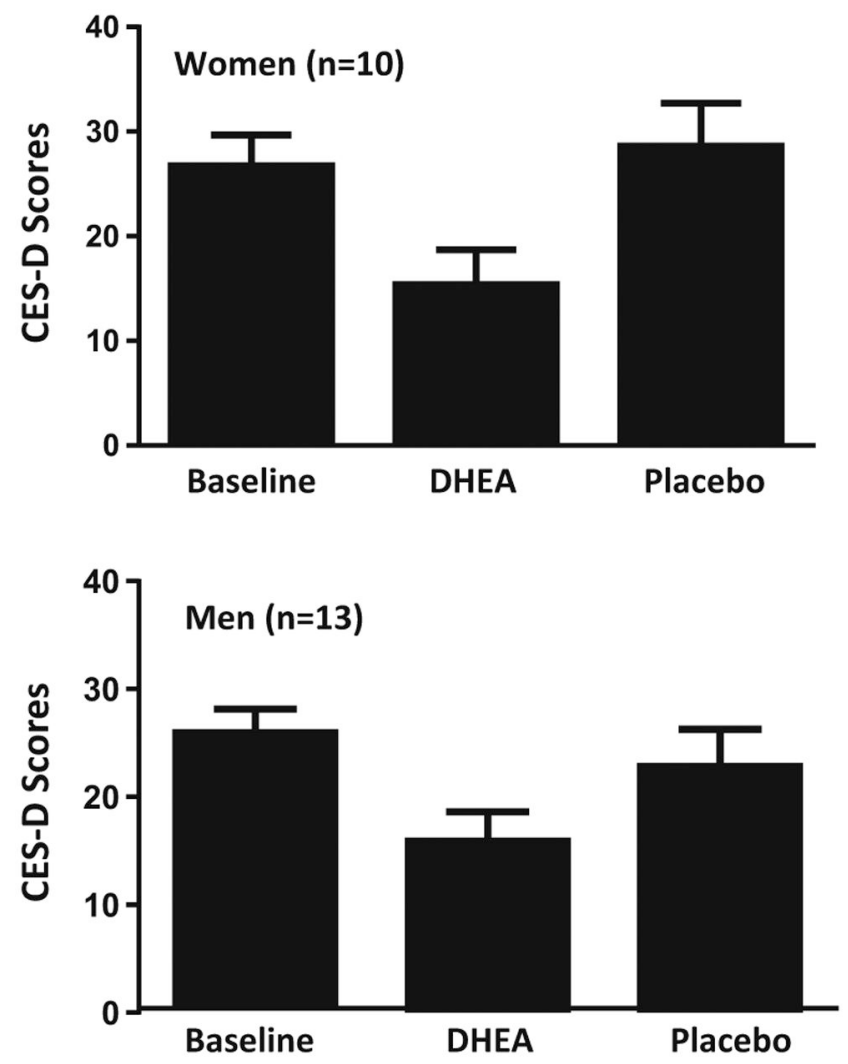

Fig. 1.

CES-D scores in women and men with midlife-onset depression treated with DHEA-mean (SE). Men and women with midlife-onset depression showed a significant decrease in CESD scores after 6 weeks of treatment with DHEA (ANOVA-R: treatment condition $\left.F_{2,40}=14.9, p<0.001\right)$. Men and women responded similarly to DHEA, and no significant effects of sex were observed in the response to DHEA (ANOVA-R: main effect of sex $F_{1,20}=0.2, p=$ ns, interactive effect of sex by treatment condition $\left.F_{2,40}=0.7, p=\mathrm{ns}\right)$. CES-D scores were significantly lower after 6 weeks of DHEA treatment compared with baseline $\left(t_{40}=5.6, p<0.01\right)$ and placebo treatment $\left(t_{40}=4.8, p<0.01\right)$; there was no difference in CES-D scores between placebo treatment and baseline $\left(t_{40}=0.4 p=\mathrm{ns}\right)$ 

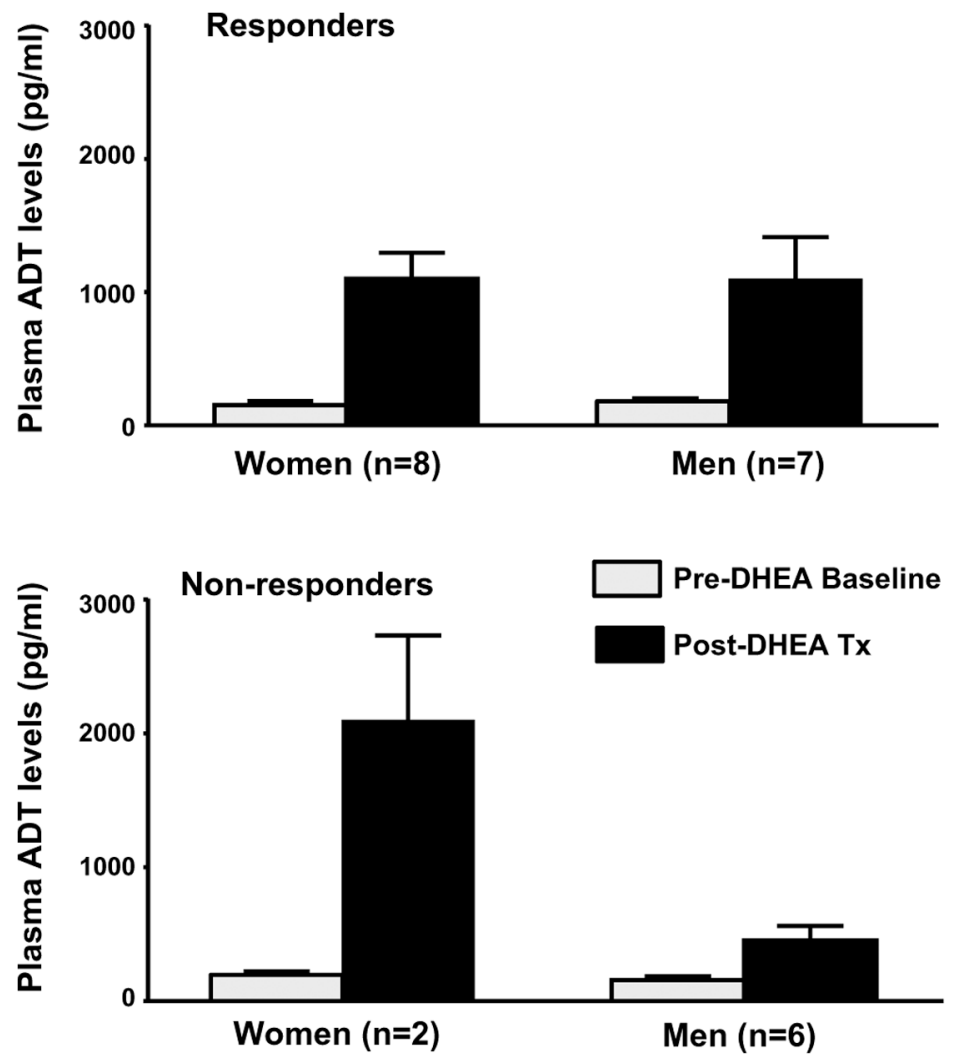

Fig. 2.

Plasma androsterone (ADT) levels in women and men with midlife-onset depression treated with DHEA: association between mood response characteristics and plasma ADT-Mean (SE). After DHEA treatment, both men and women who met our criteria for mood response had a significant increase in ADT plasma levels from baseline to the end of DHEA treatment that was of a similar magnitude $\left(t_{19}=4.4, p<0.01\right.$, both comparisons). There was no significant difference in week 6 DHEA plasma ADT levels between men and women $\left(t_{38}=0.03, p=\mathrm{ns}\right)$. In contrast, non-responding men did not show a significant increase in week 6 of DHEA treatment compared with baseline $\left(t_{19}=1.2, p=\mathrm{ns}\right)$ and non-responding women showed a greater increase than women responders (women responders compared with non-responders, $t_{38}=3.0, p<0.05$ ); however, there were only two women nonresponders. In both men and women, plasma ADT levels at baseline prior to treatment were not significantly different between responders and non-responders (women: $t_{38}=0.13$; men: $t_{38}=0.08 ; p=\mathrm{ns}$ for both comparisons) 


\section{Table 1}

\section{Sample characteristics}

\begin{tabular}{lll}
\hline Characteristic & Men $(\boldsymbol{n = 1 3})$ & Women $(\boldsymbol{n = 1 0})$ \\
\hline Age (years) (mean, SD) & $52.3(7.5)$ & $50.5(4.4)$ \\
Age range (years) & $42-63$ & $43-57$ \\
Current minor depression & $7(54)$ & $2(20)$ \\
Current major depression & $6(46)$ & $8(80)$ \\
History of major depression & $9(69)$ & $5(10)$ \\
BMI (mean, SD) & $25.1(3.1)$ & $23.9(2.0)$ \\
Weight (kg) (mean, SD) & $79.7(10.3)$ & $68.8(8.8)$ \\
CES-D score at baseline (mean, SD) & $25.9(8.1)$ & $26.7(9.2)$ \\
\hline
\end{tabular}

Unless otherwise indicated, data are expressed as number (percentage) of subjects. Reproductive status was characterized but was not a selection criterion. Women were grouped into premenopausal, perimenopausal, and postmenopausal phases of reproductive life according to the Stages of Reproductive Aging Workshop criteria (Soules et al. 2001). All participants met criteria for a current major or minor depression, as determined by the administration of the Structured Clinical Interview (SCID) for DSM IV. All participants were in good health, as evidenced by normal results on physical examinations and laboratory tests (for blood cell counts, electrolytes, liver enzymes, kidney function, and thyroid function). With the exception of one participant, who received a proton-pump inhibitor as a treatment for gastroesophageal reflux, all participants were medication free. In addition, all women had normal mammograms and gynecological examinations, and all male subjects had normal prostate-specific antigen levels

$B M I$ body mass index (weight in kilograms divided by the square of height in meters), CES-DCenter for Epidemiologic Studies Depression Scale 


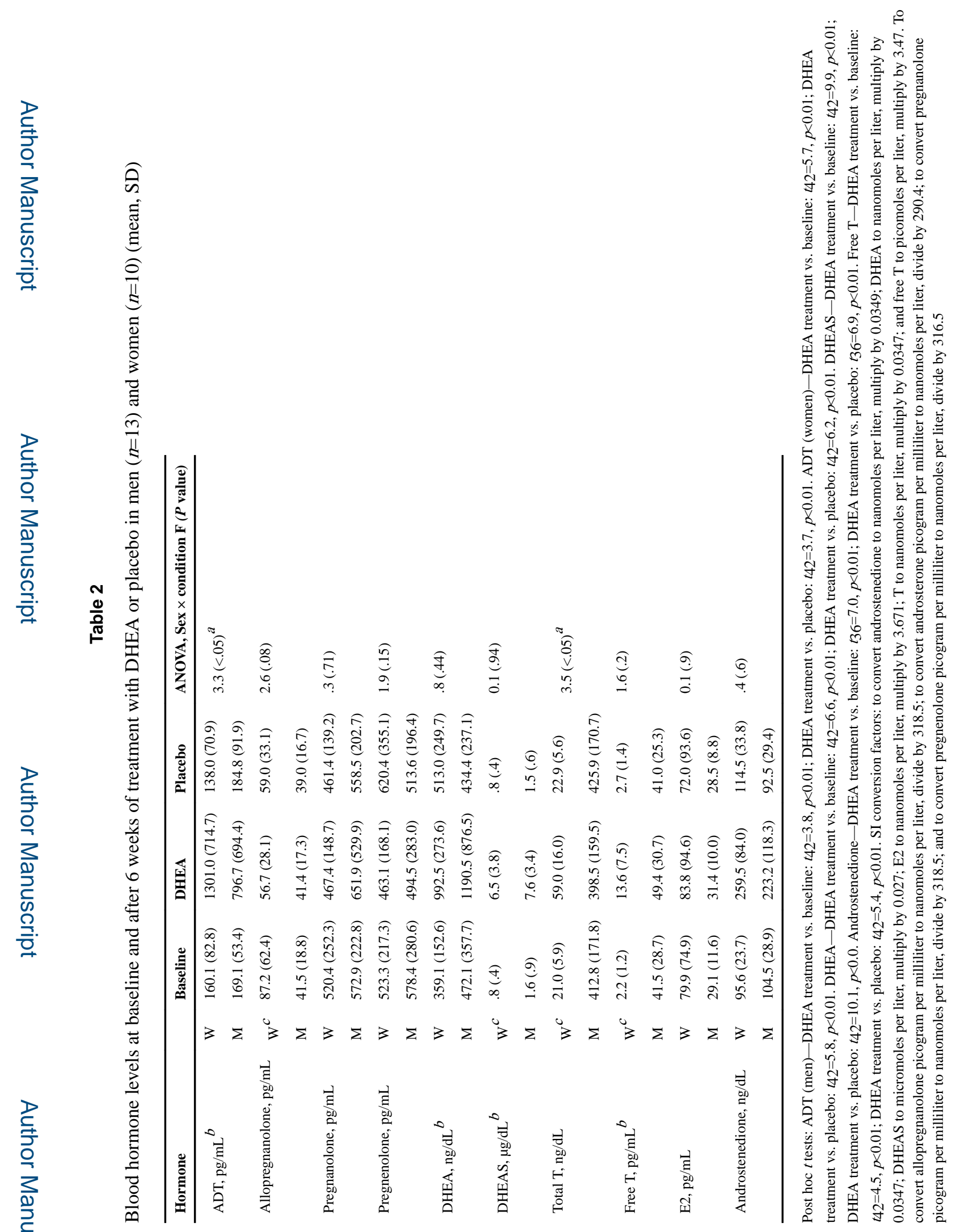

Psychopharmacology (Berl). Author manuscript; available in PMC 2018 December 28. 


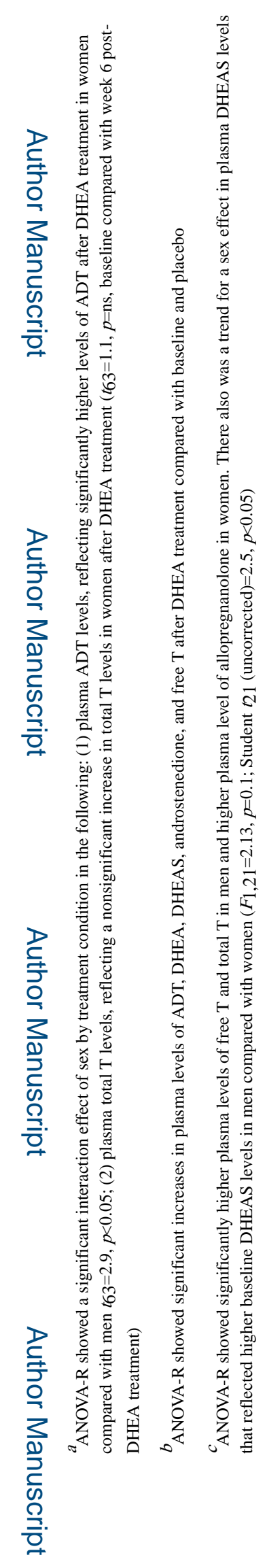

Psychopharmacology (BerI). Author manuscript; available in PMC 2018 December 28. 